\title{
Paediatric Perioperative Life Support: Safety in the Fast Lane
}

\author{
Lucy Chan* \\ Paediatric Anaesthesiologist, University Malaya, Malaysia
}

*Corresponding author: Lucy Chan, Paediatric Anaesthesiologist, University Malaya

Medical Centre, University Malaya, Malaysia, Tel: 60132815097 Email:

lucyc@ummc.edu.my

\section{Editorial}

The mission of WHO Patient Safety which declares to "facilitate sustainable improvements in patient safety and managing risks to prevent patient harm", involves all health care professionals. The anesthesiologist's workplace varies from region to region and the burden of providing safety rests substantially on their shoulders to respond adequately and competently using existing resources.

In the 1907s a dedicated group of physicians involved in the emergency care of children in North America, pioneered solutions regarding critically ill children. With that commitment, Paediatric Advanced Life Support (PALS) has evolved to be the benchmark for resuscitation training in paediatric clinical disciplines world-wide, The AHA's PALS Course has been updated with new science [1]. Of equal relevance in resuscitation medicine, the European Resuscitation Council (ERC) has published guidelines for paediatric life support [2]. ERC acknowledges that flexibility is required in crisis management due to differences in infrastructure support. Paediatric anaesthesiologists will benefit from reviewing the different scenarios that endanger the safety of a child under their care.

Many countries implement systems to gather data on critical incidents in the OT. In 1980s the Australian Patient Safety Foundation embarked on a noteworthy project known as Australian Incident Monitoring Study (AIMS). Analysis of the first 4000 critical incidents in AIMS resulted in a crisis management manual that offers a concise approach to unexpected situations during anaesthesia [3]. It contains clear algorithms to guide decision-making for all patients at risk of harm.
While the emphasis of PALS training dwells on paediatric medical critical life-threatening events and on first-responder's resuscitation, a different paediatric perioperative strategy is inevitable for understanding and competence in the management of critical incidents in OT. Awareness and comprehension of the common perioperative paediatric arrest scenarios will raise the bar on the safety and quality in the service of paediatric anaesthesia. The Paediatric Asian Society of Paediatric Anaesthesia (ASPA) founded in 2000 has an expert panel of paediatric anaesthesiologists. Its Paediatric Perioperative Life Support programme reaches out to major hospitals in the Asian region. Teaching and training include key topics, skills workshops and interactive case discussions.

The collaboration between medical science and technology has influenced training of doctors in a virtual medium. Australia conducts the Paediatric Anaesthesia Crisis Management program in their simulation centre through ANZCA. In North America, there are regular workshops on Simulation for Paediatric Anaesthesia Course Syllabus (based on ACRM principles). A valid grading system that tests on the management of simulated emergency events is available to justify the fitness of a doctor to act proficiently alone in the OT [4]. To support prompt decision-making, a mobile Apps "Pedi Crisis", initiated by the Children's Hospital of Philadelphia, has algorithms in at least 24 critical events.

Three independent factors increase the risk in general anaesthesia, namely, ASA status, emergency surgery and children under 1 year of age. The risk of critical non-fatal incidents confined specifically to children varies from 


\section{Anaesthesia \& Critical Care Medicine Journal}

$<0.5 \%$ to $8.9 \%[5,6]$. Although multiple factors often coexist to destabilize the child in anaesthesia, three categories with the highest risk include airwayrespiratory, medications and cardiovascular. The risk triples or quadruples in infants compared to older children. Basic training can reduce preventable nearmisses especially in airway management. Apart from nonpreventable adverse drug reactions, reduction of medication errors among nurses and doctors need to be addressed. In their Policy Statement, the American Academy of Pediatrics that provides Board Certification in the subspecialty of Paediatric Anaesthesia, also approves for $<2$ years old designated as increased anaesthesia risk.

PALS continues to provide a central role in paediatric life support systems even though cardiac arrest is rare in children. However, compared to cardiac arrest, a non-fatal critical incident is not uncommonly experienced by anaesthesiologists. Regular round-table discussions on problematic paediatric cases and development of local audit systems are necessary as learning instruments in the continuous medical education for safety in children in the perioperative period. Paediatric anaesthesiologists should evaluate their local support systems and take a proactive stand, such as, guiding their trainees to access the wealth of resources relevant to the safety of children under their care.

\section{References}

1. Robert W Neumar, Michael Shuster, Clifton W Callaway, Lana M Gent, Farhan Bhanji, et al. (2015) Circulation. Guidelines 2015/CPR, ECC 132 (18).
2. European Resuscitation Council Guidelines for Resuscitation 2015: Section 6. (2015) Paediatric life support. Ian K. Maconochie, Robert Bingham, Christoph Eich, Jesús López-Herce, Antonio Rodríguez-Núñez, Patrick Van de Voorde on behalf of the Paediatric life support section Collaborators (Koenraad G. Monsieurs [Eds.], Resuscitation pp: 223248.

3. Runciman WB, Kluger MT, Morris RW, Paix AD, Watterson LM, et al. (2005) Crisis management during anaesthesia: the development of an anaesthetic crisis management manual. Qual Saf Health Care 14(3): e1.

4. Everett TC, Ng E, Power D, Marsh C, Tolchard S, et al. (2013) Managing Emergencies in Paediatric Anaesthesia global rating scale is a reliable tool for simulation-based assessment in pediatric anesthesia crisis management. Paediatr Anaesth 23 (12): 11171123.

5. Ji-Hyun Lee, Eun-Kyung Kim, In-Kyung Song, Eun-Hee Kim, Hee-Soo Kim, et al. (2016) Critical incidents, including cardiac arrest, associated with pediatric anesthesia at a tertiary teaching children's hospital. Pediatric Anesthesia 26(4): 409-417.

6. Raylene Dias, Nandini Dave, Swapna Chiluveru, Madhu Garasia (2016). Critical incidents in paediatric anaesthesia: A prospective analysis over a 1 year period. Indian J Anaesth 60(11): 801-806.

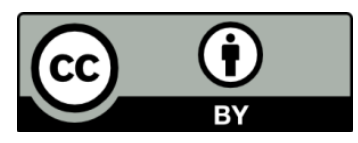

